\title{
Conversión de un ensayo de progenies de Pinus greggii var. greggii a huerto semillero mediante eigen-análisis
}

\author{
Converting a progeny test of Pinus greggii var. greggii into a seed orchard \\ based on Eigenanalysis
}

\author{
Benito Natalio Gutiérrez Vázquez ${ }^{a *}$, Eladio Heriberto Cornejo Oviedo ${ }^{\mathbf{b}}$, Alejandro Zermeño González ${ }^{\mathbf{c}}$, \\ Salvador Valencia Manzo ${ }^{b}$, Rosalinda Mendoza Villarreal ${ }^{d}$ \\ *Autor de correspondencia: anstituto Nacional de Investigaciones Forestales, Agrícolas y Pecuarias (INIFAP), México, \\ tel/fax 01 (951) 5215502 y 5216044 ext. 131, gutierrez.benito@inifap.gob.mx \\ bUniversidad Autónoma Agraria Antonio Narro, Departamento Forestal, México. \\ 'Universidad Autónoma Agraria Antonio Narro, Departamento de Riego y Drenaje, México. \\ dUniversidad Autónoma Agraria Antonio Narro, Departamento de Ciencias Básicas, México.
}

\begin{abstract}
SUMMARY
The study was conducted on a progeny test of Pinus greggii var. greggii established at Los Tarihuanes, Arteaga, Coahuila, Mexico. The objective was to convert a progeny test of Pinus greggii var. greggii into a seed orchard by simulating a silvo-genetic thinning and a tree selection through a selection index based on a principal components (CP) analysis. The selection index method based on the Eigenanalysis (i.e., principal components) allowed the selection of the best seed trees. The variables used for the construction of the selection index (basal diameter, normal diameter, volume of Pressler, total height, canopy diameter, and diameter of branches) explained $80.5 \%$ out of the total variance. The application of low and geometric thinning simulations with the Software Stand Visualization System (SVS) allowed the design of the seed orchard. The use of the SVS facilitated the design of the area as well as the visualization of the spatial distribution for each tree. Trees density in the seed orchard is within the range of densities used in other related studies.
\end{abstract}

Key words: Pinus greggii, principal component, index of selection, seed orchard.

\section{RESUMEN}

El presente trabajo se realizó en un ensayo de progenie de Pinus greggii var. greggii establecido en el predio particular Los Tarihuanes, Arteaga, Coahuila, México. El objetivo fue convertir un ensayo de progenies de Pinus greggii var. greggii a huerto semillero mediante la simulación de aclareos silvo-genéticos y la selección de árboles a través de un índice de selección basado en eigen-análisis. El índice de selección basado en eigen-análisis permitió seleccionar los mejores árboles semilleros. Las variables usadas para la construcción del índice (diámetro normal, diámetro basal, diámetro de copa, volumen de Pressler, altura y diámetro de ramas) explicaron el $80,5 \%$ de la varianza. Los aclareos por lo bajo y geométrico simulados con el Software Stand Visualization System (SVS) permitieron el diseño del huerto semillero. El uso del SVS facilitó el diseño del área visualizando la distribución espacial para cada uno de los árboles. La densidad de los árboles que integraron el huerto semillero está dentro del rango de las densidades utilizadas en otros estudios.

Palabras clave: Pinus greggii var. greggii, eigen-análisis, índice de selección, huerto semillero.

\section{INTRODUCCIÓN}

En México las prácticas de mejoramiento genético forestal se orientan al cultivo en bosques naturales con arbolado que presenta las mejores características fenotípicas. Se aplican diferentes sistemas y tratamientos silvícolas que, en los mejores casos, aseguran únicamente la regeneración de los bosques sin la certeza de que el renuevo presente una ganancia genética efectiva (Azamar et al. 2000). El suministro insuficiente de semilla es el principal factor limitante para el desarrollo de los programas de plantación (Granhof 1991). En los programas de mejoramiento genético, la información de la primera generación de los individuos de la población es puramente fenotípica. Sin embargo, a medida que se avanza en el programa, es indispensable utilizar metodologías de evaluación más eficientes (Torres 2000).

Pinus greggii var. greggii Engelm. ex Parl. es una especie nativa de la región, de rápido crecimiento, precoz en su floración y la mayoría de sus poblaciones está seriamente amenazada por factores naturales y antropogénicos que provocan el aislamiento y la reducción de sus poblaciones 
(López et al. 1993). Al realizar un estudio de variación isoenzimática en diez poblaciones naturales de Pinus greggii, seis de ellas del Norte, incluida la población de Los Lirios, Arteaga, Coahuila, México, Ramírez et al. (1997) indican que existe una reducción de la diversidad genética de esta especie debido a la tendencia de los alelos hacia el monomorfismo y a que las poblaciones de la especie se encuentren en áreas restringidas y aisladas entre sí. Además, muchas de estas poblaciones muestran una alta prioridad para la conservación de genes y la existencia de altas diferencias entre poblaciones y una baja variabilidad dentro de poblaciones. Por lo cual, es de suma importancia rescatar aquellos individuos valiosos que presenten las mejores cualidades de acuerdo a los objetivos de los programas de mejoramiento y realizar su adecuada conservación ex-situ.

En enero de 1994 se estableció un ensayo de progenies de Pinus greggii var. greggii en el predio particular Los Tarihuanes, Arteaga, Coahuila, México. El ensayo tiene el objetivo de seleccionar y rescatar los genotipos valiosos que mejor se adaptaran a las condiciones semiáridas de la región y presentaran el mejor crecimiento y desarrollo. Lo anterior debido a la amenaza que sufre la población por factores antropogénicos y además a la necesidad de contar con individuos aptos para los programas de reforestación en las zonas semiáridas y de bajas temperaturas que permitan obtener altos índices de sobrevivencia en campo.

En la actualidad existen varios métodos para el mejoramiento genético simultáneo de varios caracteres y los tres de mayor importancia son: selección en tándem, selección simultánea de caracteres independientes e índice de selección (IS). Los IS permiten separar genotipos con base en la evaluación simultánea de varios caracteres (Zobel y Talbert 1988). En el mejoramiento de plantas, los índices de selección ayudan a seleccionar los mejores individuos para el siguiente ciclo de mejoramiento en base a los valores fenotípicos observados para varias características de cada candidato individual (Cerón y Sahagún 2005). El IS comúnmente utilizado en los programas de mejoramiento por selección fue definido por Smith (1936) como una combinación lineal de los valores fenotípicos de los caracteres de interés y según Hazel (1943) es un criterio para medir el mérito neto de mejoramiento de las unidades de selección. Para Falconer (1981) el IS es el mejor predictor lineal sesgado del valor de mejoramiento de la unidad de selección y toma la forma de la regresión múltiple del valor de mejoramiento sobre todas las fuentes de información. Estos IS requieren una gran cantidad de información que incluyen estimaciones de las varianzas y covarianzas de los valores fenotípicos y genotípicos, y para cada carácter el peso económico de su valor genotípico. Por esta razón muchos fitomejoradores no utilizan estos IS en el mejoramiento de plantas (Lande 1992).

La aplicación del índice de selección es de dos tipos: uno es mejorar una sola característica y para incrementar la eficiencia de selección, es posible incluir información en los IS sobre los rasgos relacionados con la característica de interés (Wei et al. 1996, Falconer y Mackay 1997). El otro es la mejora de múltiples rasgos que requiere de la asignación de pesos económicos respectivos a los diferentes rasgos.

Cerón y Sahagún (2005) y Cerón et al. (2006) derivaron un método para construir índices de selección basados en eigen-análisis con base en el primer componente principal asociado solamente a la matriz de covarianzas fenotípicas y este nuevo método fue superior en un caso específico al de Smith (1936). El nuevo método denominado por Cerón et al. (2006) ESIM (Eigen Selection Index Method) permite estimar de manera más fácil y rápida los coeficientes de los componentes del IS. Además proporciona una mayor respuesta a la selección y no requiere ponderaciones económicas, ni estimaciones de varianzas y covarianzas genotípicas. Asimismo, Cerón et al. (2008b) desarrollaron un índice de selección restrictivo basado en eigen-análisis, denominado RESIM (Restricted Selection Index Method Based on Eigenanalysis). La principal ventaja del RESIM sobre los índices de selección restrictivos y no restrictivos tradicionales es que se conocen las propiedades estadísticas de la muestra, y la respuesta de selección es igual o mayor que los estimados por los índices de selección restrictivos tradicionales no requiere de pesos económicos y puede ser usado en aplicaciones prácticas cuando las características necesitan ser desarrolladas simultáneamente. Además, Cerón et al. (2008a) desarrollaron un índice de selección molecular basado en eigen-análisis denominado MESIM (Molecular Eigen Selection Index Method), a partir de la selección asistida por marcadores que maximiza la respuesta de selección mediante la combinación de información de marcadores moleculares ligados a los rasgos QTLs (Quantitative Trait Locus o Locus para Carácter Cuantitativo) y valores fenotípicos de los rasgos de los individuos de interés. Al analizar los resultados encontraron que el MESIN reveló que la media genotípica y la respuesta de selección esperada fueron iguales o superiores para cada característica que en los obtenidos por el MSI tradicional.

En la actualidad el uso del eigen-análisis en el mejoramiento genético forestal es mínimo, como en la selección de árboles semilleros de Pinus greggii y la conversión de un ensayo de procedencias a un rodal semillero (Cornejo et al. 2009). Sin embargo, no existen evidencias de estudios reportados sobre el establecimiento de huertos semilleros con base en eigen-análisis. Es evidente la necesidad de métodos que reduzcan los costos y el trabajo para construir índices de selección, eliminen los posibles sesgos en la estimación de los coeficientes del IS introducidos por las estimaciones de las varianzas y covarianzas de los valores genotípicos y los que, muy probablemente, conllevan las determinaciones subjetivas de las ponderaciones económicas de los valores genotípicos (Cerón y Sahagún 2005). 
El objetivo del estudio fue convertir un ensayo de progenies de Pinus greggii var. greggii a huerto semillero, mediante la simulación de aclareos silvo-genéticos y la selección de árboles a través de un índice de selección basado en eigen-análisis en el predio particular Los Tarihuanes, en Arteaga, Coahuila, México.

\section{MÉTODOS}

Descripción del área experimental. El ensayo de progenies de Pinus greggii var. greggii se encuentra en las coordenadas $25^{\circ} 22^{\prime} 13^{\prime \prime} \mathrm{N}$ y $100^{\circ} 36^{\prime} 22^{\prime \prime} \mathrm{O}$, con una altitud aproximada de $2.400 \mathrm{~m}$ s.n.m. Se utilizó un diseño en bloques completamente al azar con cuatro bloques colocados perpendicularmente respecto de la pendiente, con cuatro plantas por parcela. El ensayo de progenies se constituyó de 18 árboles maternos procedentes de Los Lirios, Arteaga, Coahuila, México, y un testigo procedente del Rincón del Molino, Cerro viejo, Bella Unión, Coahuila, México. La edad de las plantas al momento de la plantación fue de 40 a 42 meses y el testigo de 48 meses; se plantó en cepa común con una distribución en tresbolillo con $3 \mathrm{~m}$ de espaciamiento. Se utilizaron 283 plantas útiles en total y 76 de borde. De acuerdo con los datos registrados en la estación meteorológica $\mathrm{N}^{\mathrm{o}}$ 05-033, San Antonio de las Alazanas, la fórmula climática del área es: $\mathrm{Cb}\left(\mathrm{x}^{\prime}\right)$ (e') $\mathrm{g}$, que corresponde a un clima templado; y de acuerdo a la estación meteorológica de Jamé, se registra una temperatura media anual de $12,9^{\circ} \mathrm{C}$, con una precipitación media anual de 428,6 mm (García 1987).

Variables evaluadas. Las variables que se evaluaron para construir el índice de selección fueron: la altura total del árbol, el diámetro basal y el diámetro normal (a 1,30 m a partir del suelo). Para el cálculo de los crecimientos relativos se consideraron la altura total inicial y el diámetro basal inicial medidos en 1992. La altura total, el diámetro basal y el diámetro normal finales se evaluaron en diciembre de 2006.

También se midieran el diámetro de copa, el diámetro de las tres ramas más gruesas que se encontraban en cada uno de los tres primeros verticilos por arriba del diámetro normal, el ángulo de inclinación de las ramas con respecto al fuste. Además, se calificó la rectitud del fuste usando números del uno al cuatro como sigue: el 1 para árboles rectos, 2 para árboles inclinados, 3 para árboles torcidos y 4 para árboles bifurcados. Se realizó un conteo directo desde la base del árbol hasta la yema apical, para las variables número de verticilos y número de ramas por verticilo, considerando como verticilo todos aquellos que tenían dos o más ramas. En el caso de las ramas por verticilo se obtuvo un promedio de las ramas de cada verticilo.
Se calcularon los crecimientos relativos en altura total (CRA) y en diámetro basal (CRDB), ambas mediante la ecuación 1.

$$
C R A=\frac{L n_{A_{f}}-L n_{A_{i}}}{T_{f}-T_{i}}
$$

Donde: $\mathrm{CRA}=$ crecimiento relativo en altura; $\mathrm{Ln}=$ logaritmo natural; $A_{\mathrm{f}}=$ altura final; $A_{\mathrm{i}}=$ altura inicial; $\mathrm{T}_{\mathrm{f}}=$ tiempo final (20 de diciembre de 2006); $\mathrm{T}_{\mathrm{i}}=$ tiempo inicial (1992).

El volumen de Pressler (Romahn 1991) se calculó con la ecuación 2.

$$
V=\frac{2}{3} *(A * h)
$$

Donde: $\mathrm{V}=$ volumen de Pressler $\left(\mathrm{m}^{3}\right) ; \mathrm{a}=$ área seccional transversal del tronco a $1,30 \mathrm{~m}$ de altura $\left(\mathrm{m}^{2}\right) ; \mathrm{h}=$ altura de Pressler $=d+1,30 m+0,65 m ; d=$ distancia entre diámetro normal y la sección con diámetro igual a la mitad del diámetro normal (m).

Se estimó un cociente de ramas (Cocirama) que se obtuvo con el diámetro a la base del árbol y el promedio del diámetro de las ramas más gruesas que se encontraban en los tres verticilos por arriba del diámetro normal.

$$
\text { Cocirama }=\frac{D B}{\text { Dpromr }}
$$

Donde: Cocirama $=$ cociente de ramas; $\mathrm{DB}=$ diámetro basal; Dpromr $=$ diámetro promedio de las ramas.

Todas las variables se estandarizaron con la finalidad de cumplir con el procedimiento de los análisis multivariados (SAS Institute Inc. 1999); para la estandarización del ángulo de las ramas se realizó una transformación angular y para el resto de las variables se realizó con la ecuación 4.

$$
Z i j=\left[\frac{X i j}{S j}\right]^{2}
$$

Donde: $\mathrm{Z} i j=$ nuevo valor estandarizado $; \mathrm{X} i j=$ valor de la observación $i$ en la variable $j ; \mathrm{S} j=$ desviación típica o estándar de los valores de la variable $j$.

Eigen-análisis. Para determinar el índice de selección se utilizó el método de eigen-análisis (Cerón y Sahagún 2005), denominado como ESIM por Cerón et al. (2006). Se realizó un eigen-análisis con el Statistical Analysis System (SAS) versión 9.1, utilizando el procedimiento PRINCOMP. El proceso de análisis de los datos se realizó mediante un proceso iterativo hasta encontrar el menor número de variables que explicaron la mayor variación total 
con el primer componente principal (CP). Los elementos del vector característico del primer $\mathrm{CP}$ determinaron la proporción con que los caracteres respectivos contribuyen al nuevo índice. La selección de los mejores árboles se realizó sobre la base de la correlación entre las variables dasométricas y de forma de copa. Se eliminaron las variables que no presentaron una alta correlación dentro del $\mathrm{CP}$ y se seleccionaron solamente las variables que mejor se asociaron dentro del CP.

Para agrupar los árboles de acuerdo a las variables resultantes de la reducción de los $\mathrm{CP}$ se utilizó el procedimiento CLUSTER de SAS para lo cual se probó el método Pseudo Ward Pseudo. Se seleccionó el grupo que presentara los valores más altos del criterio cúbico de agrupamiento (CCA) y de la pseudo F estadística (estadístico que mide la separación entre todos los grupos del nivel actual). Cada árbol se identificó con un código diferente para ubicar en qué grupo se encontraba.

Simulación de aclareos silvo-genéticos. Una vez obtenido el índice de selección se realizó la validación de los árboles en campo, verificando cuáles fueron las variables de los árboles por las que se habían integrado a cada grupo. En la validación se observaron espacios dentro del ensayo debido a la mortalidad (38,9\%), lo que manifiesta un primer filtro de adaptación, sobreviviendo aquellos árboles resistentes a las bajas precipitaciones y altas temperaturas. Los árboles presentes en el centro del ensayo no presentaban un tallo fuerte, por lo que se consideró dejar árboles no selectos de protección en los lugares donde los vientos ocurren con mayor frecuencia y éstos serán eliminados cuando cumplan con el propósito. Para la simulación de los aclareos, se utilizó el sistema de visualización de rodales (SVS) Versión 3,36 (McGauhuey 2000) con el cual se diseñó el huerto semillero, representando el ensayo de progenies inicial y cada una de las simulaciones de los aclareos.

Los criterios que se consideraron en la simulación fueron el aclareo geométrico, el espaciamiento y la representatividad de las familias; así como las variables del índice de selección y el micrositio. Para llevar a cabo el diseño del huerto semillero se consideró la representatividad de las familias para obtener mayor variación, lo que consistió en no dejar en una misma parcela árboles de la misma familia y dejando el mayor número posible de familias representadas. El software SVS permitió realizar aclareos por lo bajo y geométricos de tal manera que existiera suficiente espacio para el movimiento de maquinaria para el futuro manejo del huerto.

El índice de selección fue el primer criterio para la selección de árboles semilleros. El micrositio se consideró como la posición que ocupaban los árboles dentro del ensayo. La posición describió si el árbol se encontraba en el centro o en la orilla del ensayo. La importancia del micrositio radicó en la cantidad de luminosidad recibida por los árboles y que los árboles que se encontraban en el centro del ensayo presentaban fustes menos resistentes a los vientos y acículas menos tolerantes al sol, que al quedar parcial o completamente expuestos al sol entran en estrés, provocando su muerte.

El aclareo geométrico consistió en realizar aclareos de tal manera que existiera una equidistancia entre los árboles, realizando un primer aclareo por lo bajo que permitió eliminar aquellos árboles que se encontraban por debajo del dosel y que eran árboles indeseables. En el espaciamiento y el aclareo geométrico se utilizó una distancia mayor o igual a $4 \mathrm{~m}$, distancia adecuada para la movilidad de maquinaria dentro del huerto.

\section{RESULTADOS}

Eigen-análisis. El diámetro basal, diámetro normal, diámetro de copa, volumen de Pressler, altura total y el diámetro de ramas integraron el índice de selección, el cual explicó el $80,5 \%$ de la varianza con el primer componente principal (CP). Las variables que presentaron mayor contribución relativa a la variación total fueron las variables dasométricas. El diámetro basal, diámetro normal y el volumen de Pressler mostraron una mayor correlación con el primer CP que la altura total, diámetro de copa y el diámetro de ramas (cuadro 1). El índice de selección resultó ser muy efectivo porque los árboles elegidos por el modelo, efectivamente fueron los mejores en campo.

Cuadro 1. Eigenvectores de las variables que integraron el índice de selección de Pinus greggii var. greggii. of Pinus greggii.

Eigenvectors for variables included into the selection index

\begin{tabular}{lc}
\hline \multirow{2}{*}{ Variable } & $\begin{array}{c}\text { Componente principal } \\
\text { (Varianza explicada) }\end{array}$ \\
\cline { 2 - 2 } & $1(80,54 \%)$ \\
\hline Diámetro basal & 0,4383 \\
Diámetro normal & 0,4434 \\
Volumen de Pressler & 0,4392 \\
Altura total & 0,3875 \\
Diámetro de copas & 0,3823 \\
Diámetro de ramas & 0,3490
\end{tabular}

Se encontró que el método de pseudo Ward fue el que mejor agrupó a los árboles en dos grupos de acuerdo al índice de selección, con valores más altos del criterio cúbico de agrupamiento $(\mathrm{CCA})=14,3$ y de la pseudo $\mathrm{F}$ con un valor de 1.069. Los árboles del grupo 2 mostraron valores promedios más altos que los de grupo 1 , en variables como diámetro basal $(17,3$ vs 12,8 cm), diámetro normal $(12,2$ vs $8,3 \mathrm{~cm})$, altura total $(7,01$ vs $5,3 \mathrm{~m})$ y volumen de Pressler $\left(0,05\right.$ vs $\left.0,02 \mathrm{~m}^{3}\right)$. Los árboles del grupo 2 fueron los candidatos a formar el huerto semillero.

Simulación de aclareos silvo-genéticos. Al convertir el ensayo de progenies a huerto semillero, se logró que 12 de las 18 familias más el testigo quedaran representadas 
dentro del huerto semillero, donde la familia 38 fue la mejor representada con 6 individuos, la familia 44 con 4 individuos y las familias $8,17,18,19$ y 42 con 3 individuos cada una. La simulación del primer aclareo se llevó a cabo por lo bajo; se eliminaron 62 árboles, es decir, el $56,4 \%$ del total de la población superviviente, con un residual de un total de 48 árboles en pie y una densidad de 137 árboles ha ${ }^{-1}$. En la simulación del segundo aclareo de forma geométrica se eliminaron 14 árboles, equivalentes al $12,7 \%$ con relación al total de la población, con un residual de 34 árboles en pie en 0,35 ha y una densidad de 97 árboles ha ${ }^{-1}$ (figuras 1 y 2).

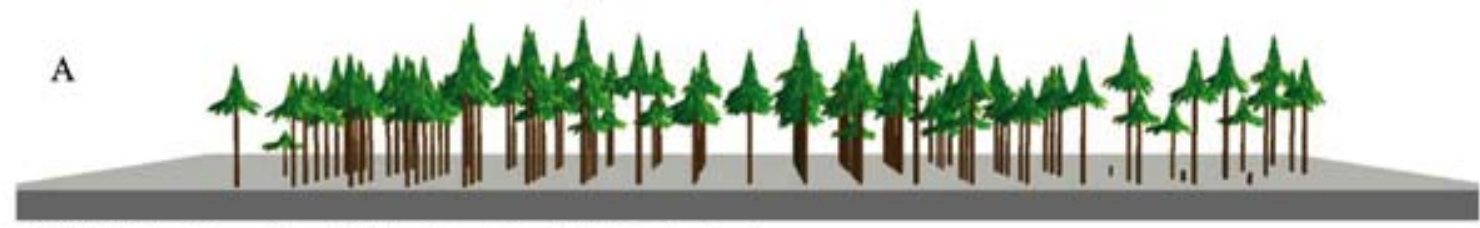

110 árboles. Superficie 0,35 ha. Densidad 314 árboles ha-1

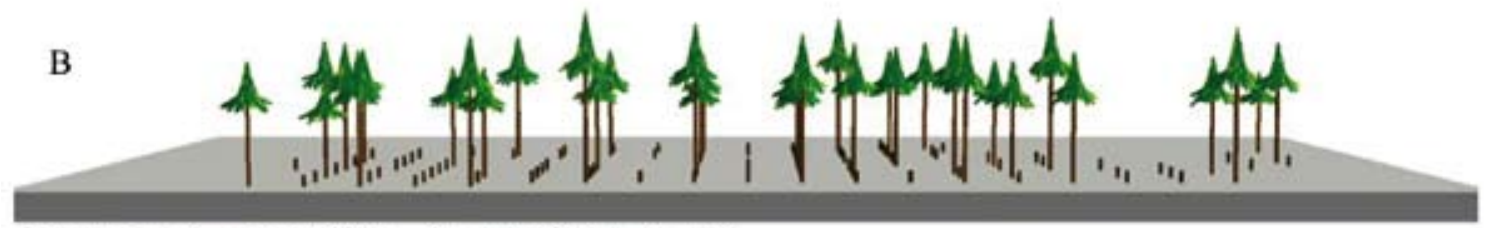

34 árboles. Superficie 0,35 ha. Densidad 97 árboles ha ${ }^{-1}$

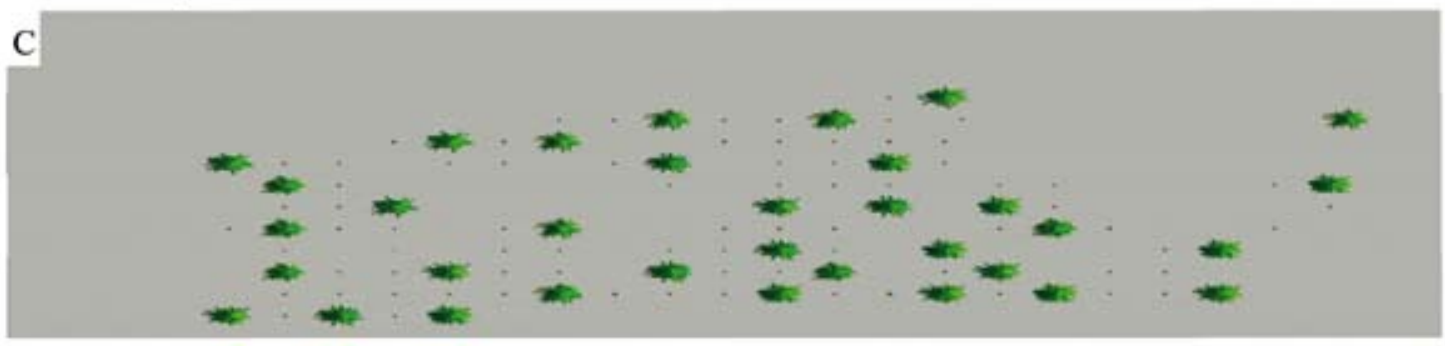

Figura 1. Ensayo de progenie de Pinus greggii (A), huerto semillero diseñado (B) y vista de las copas del huerto semillero diseñado $(\mathrm{C})$.

Progeny test of Pinus greggii. (A), Seed orchard design (B) and view of crowns of the seed orchard (C).

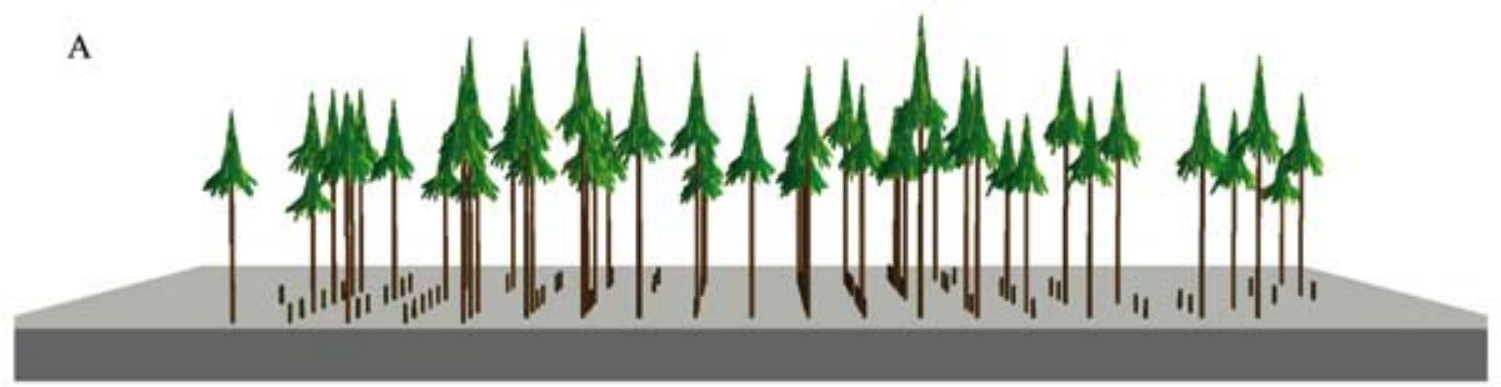

Eliminando el $56,4 \%$ del arbolado

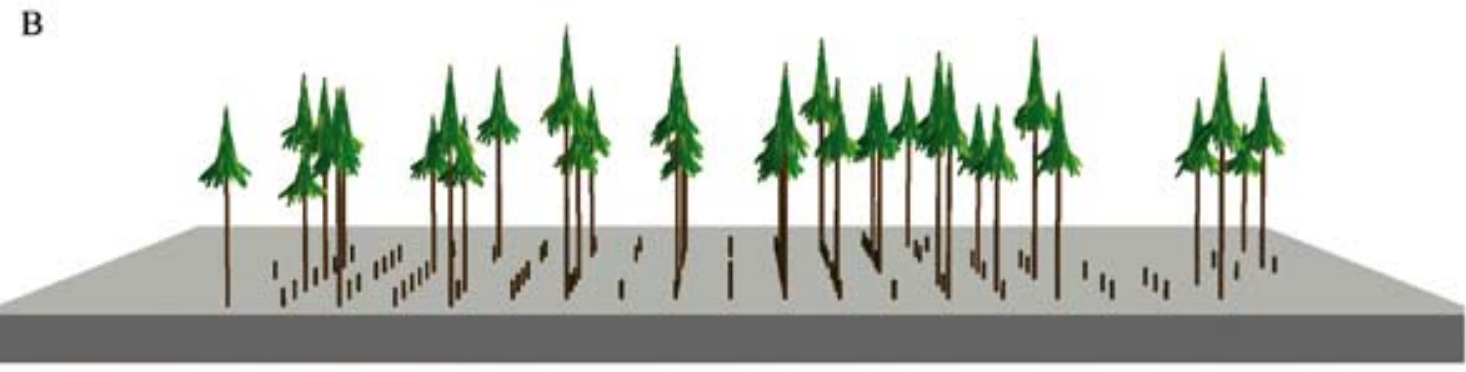

Eliminando el $12,7 \%$ del arbolado

Figura 2. Simulación de aclareos en el ensayo de progenie de Pinus greggii. Primer (A) y segundo aclareo (B). Simulation of thinning in the progeny test of Pinus greggii. first (A) and second thinning (B). 


\section{DISCUSIÓN}

Eigen-análisis. El índice de selección derivado del eigenanálisis, formado por el diámetro basal, diámetro normal, volumen de Pressler, altura total, diámetro de copa y diámetro de ramas, permitieron seleccionar 34 árboles y dejar una densidad de 97 árboles ha ${ }^{-1}$; siendo el primer componente el que mejor explica la contribución de estas variables, lo cual reafirma lo encontrado por Cerón y Sahagún (2005), Cerón et al. (2006) y Cornejo et al. (2009). El procedimiento de eigen-análisis para crear el índice de selección es sencillo y permite seleccionar los mejores árboles dentro del ensayo de progenies. Sin la necesidad de asignar pesos económicos y la estimación de la matriz de covarianzas genotípicas, ya que los índices de selección tradicionales no pueden mejorar varias características simultáneamente si los pesos económicos no están disponibles.

Al igual que Cerón et al. (2006) al construir un índice basado en el método de eigen-análisis denominado ESIM (Eigen Selection Index Method), la construcción del índice para seleccionar los mejores árboles de Pinus greggii var. greggii involucra el primer componente principal (CP), la raíz y vector característico de la matriz de covarianzas de los valores fenotípicos de los caracteres estudiados.

Las variables que integraron el índice de selección son consistentes, ya que son variables que han sido utilizadas para la construcción de otros índices, como Ladrach et al. (1977) para selección de árboles de Cupressus lusitanica Mill. en Colombia; Bridgwater et al. (1983) en la construcción de un índice de selección en Pinus taeda Schl. et Cham. en el suroeste de Georgia; Park et al. (1989) en la construcción de un índice de selección para Pinus banksiana Lamb. establecida en cuatro localidades de Brunswick, Canadá; Azamar et al. (2000) en la conversión de un ensayo de procedencias-progenies de Pinus greggii a huerto semillero en el Estado de México, México; Torres (2000) en la construcción de un índice de selección en un ensayo de progenies de Pinus radiata D. Don establecido en Chile; Valencia y Vargas (2001) en un estudio efectuado en una prueba de progenies de Pinus patula Schiede en Zacualpan, Veracruz, México; Harrand (2002) en la transformación de un ensayo de progenies a huerto semillero de Eucalyptus grandis Hill ex Maiden establecido en Argentina; Farfán et al. (2002) al evaluar la eficiencia de selección temprana de Pinus ayacahuite Ehren var. Ayacahuite establecido en Zacualpan, Veracruz, México, y Cornejo et al. (2009) al seleccionar árboles semilleros con base en un índice de selección y diseñar un rodal semillero de Pinus greggii establecido en Arteaga, Coahuila, México.

La cantidad de trabajos reportados sobre la conversión de ensayos de procedencias y progenies a huertos semilleros es muy pequeña, tal como Azamar et al. (2000) al convertir un ensayo de procedencias-progenies de Pinus greggii a huerto semillero en el Estado de México, México; Harrand (2002), al transformar un ensayo de progenies a huerto semillero de E. grandis establecido en Argentina, y Cornejo et al. (2009) al convertir un ensayo de procedencias de Pinus greggii a rodal semillero en Arteaga, Coahuila, México. Por tal motivo, es importante establecer nuevas metodologías y criterios más sencillos para llevar a cabo este tipo de trabajos.

De acuerdo a los resultados obtenidos en este trabajo, se recomienda el uso del índice de selección basado en eigen-análisis denominado ESIM (Eigen Selection Index Method), desarrollado por Cerón et al. (2006) para sus usos en la conversión de ensayos de progenies de especies forestales a huertos semilleros. Además, se recomienda el estudio de la aplicación de este índice en la selección de árboles superiores en rodales naturales porque es un índice de selección muy sencillo y permite la selección de los mejores árboles sin olvidar que el índice sólo es una herramienta, por lo cual es indispensable el uso de otros criterios que fortalezcan aún más la selección. El método de eigen-análisis es desarrollado en varias aplicaciones del mejoramiento genético, tales como Cerón et al. (2008b) al desarrollar el índice de selección restrictivo RESIM, y Cerón et al. (2008a) al construir un índice de selección apoyado en marcadores moleculares denominado MESIM (Molecular Eigen Selection Index Method) basado en un eigen-análisis.

Es ineludible tomar en cuenta que el ESIM desarrollado por Cerón et al. (2006) es afectado directamente por las varianzas genotípicas y fenotípicas de las variables, esto debido a que el método asigna más peso a las variables con varianzas fenotípicas más grandes. Además, en las características que presentan varianzas fenotípicas y genotípicas menores que puede tener alta heredabilidad, el ESIM asigna valores bajos a esta característica sin tomar en cuenta si presenta heredabilidad alta. Sin embargo, las propiedades estadísticas de los estimadores del ESIM son conocidas; al mismo tiempo, estima la ganancia de la selección entre ciclos de selección y también propone una función predictiva de la respuesta de selección del ESIM en futuros ciclos de selección.

Por lo tanto, queda de manifiesto que el método basado en eigen-análisis (i.e., componentes principales) es importante porque es una metodología simple y aplicable que permite la selección de múltiples características, y se puede aplicar en el mejoramiento genético forestal. Asimismo, es una metodología que ha mostrado una respuesta de selección mayor que los índices de selección tradicionales (Cerón et al. 2006) y no requiere ponderaciones económicas, ni estimaciones de varianzas y covarianzas genotípicas.

Simulación de aclareos. Los criterios que se consideran en la simulación de aclareos permiten realizar un análisis a detalle que explora a cada uno de los árboles seleccionados. Se consigue conservar la mayoría de las familias existentes al inicio en el ensayo, lo cual es muy importante, porque la mayoría de las poblaciones de Pinus greggii var. greggii se encuentra seriamente amenazada por factores 
naturales y antropogénicos, y existe una reducción de la diversidad genética. Con esto, se logra la conservación ex-situ adecuada de las mejores familias.

Quedó diseñado el huerto con 34 árboles en 0,35 ha, con una densidad de 97 árboles ha ${ }^{-1}$, que permite la operación mecánica de tractores y otras máquinas para el manejo futuro del huerto (Clausen 1990). La densidad utilizada en este trabajo es menor que la que manejaron Ladrach et al. (1977) en el establecimiento de un huerto semillero de Cupressus lusitanica en Colombia, con una densidad de 190 árboles ha $^{-1}$; Azamar et al. (2000) en la conversión de un ensayo de procedencias-progenies de Pinus greggii a huerto semillero en el Estado de México, México, con una densidad de 339 árboles $\mathrm{ha}^{-1}$; y Harrand (2002) en la transformación de un ensayo de progenies transformado en huerto semillero de Eucalyptus grandis Hill ex Maiden establecido en Argentina, con una densidad de 265 árboles ha ${ }^{-1}$.

Sin embargo, Zobel y Talbert (1988) recomiendan una densidad de 80 a 150 árboles ha ${ }^{-1}$ y Schmidt (1993) recomienda una densidad de 100 árboles ha ${ }^{-1}$. Por lo tanto, la densidad con la cual quedó diseñado el huerto semillero de Pinus greggii var. greggii es aceptable.

En la actualidad, en México el trabajo de Cornejo et al. (2009) es la única evidencia del uso del sistema de visualización de rodales (SVS) como herramienta dentro del estudio de bosque y plantaciones. Se recomienda el uso del SVS porque permite observar la distribución espacial de cada uno de los árboles y aumentar el nivel de detalle del análisis, logrando de esta forma una mejor comprensión del comportamiento de los árboles en las distintas situaciones existentes dentro del rodal natural o plantación. Asimismo, se realiza una mejor sistematización de los aclareos y sin duda es una herramienta importante en la conversión de ensayos de progenies a huerto semillero que, aunado al método de eigen-análisis, constituyen herramientas muy valiosas en el estudio de los recursos naturales y genéticos. Por lo tanto, se recomienda el estudio de la aplicación que puede tener el SVS no sólo en las plantaciones sino también en rodales naturales.

\section{CONCLUSIONES}

El índice de selección basado en eigen-análisis fue sencillo y permitió seleccionar los mejores árboles semilleros dentro del ensayo de progenies, sin la necesidad de asignar pesos económicos y la estimación de la matriz de covarianza genotípica.

El SVS permitió diseñar el huerto semillero al observar la distribución espacial de cada uno de los árboles, aumentar el nivel de detalle del análisis, lograr una mejor comprensión del comportamiento de los árboles y una mejor sistematización de los aclareos.

El método de eigen-análisis y el SVS son herramientas importantes en la conversión de ensayos de progenies a huerto semillero, y en el estudio de los recursos naturales y genéticos.

\section{AGRADECIMIENTOS}

A la Universidad Autónoma Agraria Antonio Narro por el apoyo para el proyecto: Ecofisiología forestal y mejoramiento genético forestal en el Campo Agrícola Experimental Sierra de Arteaga, Arteaga, Coahuila, México, clave 02.03.0207.2364; y al Consejo Nacional de Ciencia y Tecnología por la beca para realizar los estudios de maestría del primer autor.

\section{REFERENCIAS}

Azamar OM, J López, JJ Vargas, A Plancarte. 2000. Evaluación de un ensayo de procedencias-progenie de Pinus greggii y su conversión a huerto semillero. In Memorias del Primer Congreso Nacional de Reforestación. México, México. SEMARNAP-Colegio de Postgraduados Montecillo. 9 p.

Bridgwater FE, JT Talbert, S Jahromi. 1983. Index selection for increased dry weight in a young loblolly pine population. Silvae Genetica 32: 5-6.

Cerón RJJ, J Sahagún. 2005. Un índice de selección basado en componentes principales. Agrociencia 39: 667-677.

Cerón RJJ, J Crossa, J Sahagún, F Castillo, A Santacruz. 2006. A Selection Index Method Based on Eigenanalysis. Crop Science 46: 1711-1721.

Cerón RJJ, F Castillo, J Sahagún, A Santacruz, I Benítez, J Crossa. 2008a. A Molecular Selection Index Method Based on Eigenanalysis. Genetics 180: 547-557.

Cerón RJJ, J Sahagún, F Castillo, A Santacruz, J Crossa. 2008b. A Restricted Selection Index Method Based on Eigenanalysis. Journal of Agriculture, Biology and Environmental Statistics 13(4): 440-457.

Cornejo OEH, E Bucio, B Gutiérrez, S Valencia, C Flores. 2009. Selección de árboles y conversión de un ensayo de procedencias a un rodal semillero. Revista Fitotecnia Mexicana. 32(2): 87-92.

Clausen KE. 1990. Producción de semillas forestales genéticamente mejoradas. In Eguiluz PT, A Plancarte B eds. Memoria sobre Mejoramiento Genético y Plantaciones Forestales. Chapingo, México. p. 78-88.

Falconer DS. 1981. Introduction to quantitative genetics. New York, USA. Longman. 340 p.

Falconer DS, TFC Mackay. 1997. Introduction to quantitative genetics. New York, USA. Longman. 464 p.

Farfán VEG, J Jasso, J López, JJ Vargas, C Ramírez. 2002. Parámetros genéticos y eficiencia de la selección temprana en Pinus ayacahuite Ehren. var. ayacahuite. Revista Fitotecnia Mexicana 25(3): 239-246.

García E. 1987. Modificación al sistema de clasificación climática de Köppen, para adaptarla a las condiciones de la República Mexicana. México. UNAM. 245 p.

Harrand L. 2002. Utilización de "valores de cría" para la habilitación de un huerto semillero de progenies. Proyecto de Desarrollo Forestal-PPMPM Eucaliptos en Mesopotamia. Concordia, Argentina. EEA INTA. 5 p.

Hazel LN. 1943. The genetic basis for constructing selection index. Genetics 28: 476-490.

Granhof J. 1991. Mass production of improved material. Seed orchards. Lecture note $\mathrm{N}^{\mathrm{o}}$ D-8. Danida Forest Seed Centre. Denmark. 26 p. 
Ladrach W, M Gutiérrez, P Arboleda, MH García. 1977. Establecimiento de un huerto semillero de Cupressus lusitanica Mill. Informe de Investigación No. 25. Cali, Colombia. Smurfit Cartón Colombia S.A., Investigación Forestal. 16 p.

Lande R. 1992. Marker-assisted selection in relation to traditional methods of plant breeding. In Stalker HT, JP Murphy eds. Plant Breeding in the 1990's. UK. C.A.B International. p. 437-458.

López UJ, J Jasso, JJ Vargas, C Ayala. 1993. Variación de características morfológicas en conos y semillas de Pinus greggii. Agrociencia 3(1): 81-84.

McGauhuey RJ. 2000. Stand Visualization System. Version 3.36. Washington. Pacific Northwest Research Station USDAFS. $141 \mathrm{p}$.

Park YS, JD Simpson, DP Fowler, EK Morgenstern. 1989. A selection index with desired gains to rogue jack pine seedling seed orchards. Information report M-X-176. New Brunswick, Canada. Forestry Canada-Maritimes Region. 18 p.

Ramírez HC, JJ Vargas, J Jasso, G Carrillo, H Guillén. 1997. Variación isoenzimática de diez poblaciones naturales de Pinus greggii Engelm. Agrociencia 31: 223-230.

Romahn de la VCF. 1991. Relascopía. Serie de apoyo académico No. 43. Chapingo, México. Universidad Autónoma Chapingo. División de Ciencias Forestales. 133 p.
SAS Institute Inc. 1999. SAS/STAT Guide for personal computers. Version 6. Cary, NC. USA. 518 p.

Schmidt L 1993. Seed Orchards. Guidelines on establishment and management practices. Undp/FAO regional project on improved productivity of man-made forests through application of technological advances in tree breeding and propagation (ras/91/004). Philippines. $103 \mathrm{p}$.

Smith HF. 1936. A discriminant function for plant selection. Ann. Eugenics 7: 240-250.

Torres J. 2000. Evaluación genética y económica de dos ensayos de progenie de Pinus radiata D. Don a base de un índice de selección multicriterio. Ciencias Forestales 14(1-2): 1-7.

Valencia MS, JJ Vargas. 2001. Correlaciones genéticas y selección simultánea del crecimiento y densidad de la madera en Pinus patula. Agrociencia 35: 109-120.

Wei M, A Caballero, HG Hill. 1996. Selection response in finite populations. Genetics 144: 1961-1974.

Zobel BJ, JT Talbert. 1988. Técnicas de mejoramiento genético de árboles forestales. México, México. Limusa. 545 p.

Recibido: 04.08.09

Aceptado: 09.11.09 